\title{
Stochastic traffic generator for Monte Carlo load flow simulation
}

\author{
E. Pilo de la Fuente ${ }^{1,2}$, J. Jiménez-Octavio ${ }^{1}$, R. Rodriguez- \\ Pecharroman $^{1} \&$ A. Lopez Lopez ${ }^{1}$ \\ ${ }^{1}$ Universidad Pontificia Comillas de Madrid, Spain \\ ${ }^{2}$ Multitest09, Madrid, Spain
}

\begin{abstract}
This paper presents a stochastic traffic generator in which (i) the driving manner is assumed to be stochastic, (ii) random unexpected stops are considered and (iii) the stop time in the stations is also supposed to be stochastic. This simulation tool provides a large number of different driving scenarios to be used for a Monte Carlo load flow analysis. Traffic simulations algorithms are described in detail. Reference to a section of the Madrid-Barcelona high-speed line illustrates the potential of such a tool.
\end{abstract}

Keywords: power supply, train driving, stochastic simulation.

\section{Introduction}

One of the inputs required for designing the electrification in a railway system is the power to be supplied to the rolling stock in each time step, which is in itself closely related to the considered operation. Accordingly, compared to other power systems, railways are quite different: power consumptions are moving loads and change very quickly. The power consumption depends on the manner in which the trains are driven (the speed along the trip, how fast it accelerates or it brakes, etc.), the characteristics of the train (mass, running resistance, motors, etc.) and the path (slopes, speed limits, etc). Due to its complexity, computation of instant power consumption of the trains is normally performed by software tools.

When traction power consumptions have to be estimated, the characteristics of the train and the path are typically well defined. However, rail lines are normally not equipped with automatic driving systems and each driver drives his 
own way (within mandatory safety limitations). Thus, in theory, there is an infinite number of possible ways of driving, with very different power consumption spatial distributions. The driving type in which the trip is done as fast as possible, considering both the infrastructure speed and the train operational limits, is known as minimal time driving (MTD) [1].

When performing power supply system design, MTD is normally considered because: (i) it is very simple to define the manner in which trains are driven and (ii) it is mistakenly believed to be the most demanding driving mode.

This paper discusses why MTD mode does not correspond to the worst driving mode for the railways electrification and presents a simulation algorithm which combines MTD simulations and stochasticity in the operation. The final purpose of this simulation is to carry out stochastic analysis of power supply systems.

Section 2 describes the power supply system, its design and why MTD does not necessarily correspond to the worst operating conditions. Section 3 presents the simulation procedure that is proposed in this paper. Section 4 presents some results obtained with this simulation tool. Finally, Section 5 summarizes the conclusions of this work.

\section{Power supply system}

\subsection{General structure}

Figure 1 shows the general structure of power-supply systems of AC electrified railways, when they are fed from a three-phase network. Even if this paper is focused on this case, similar conclusion can be reached in other systems.

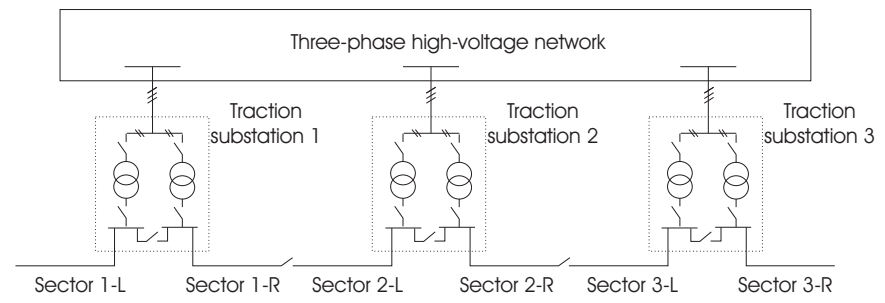

Figure 1: Structure of the power supply system.

As shown, the electrical system is divided in electrically-isolated sectors, which are fed from the three-phase network through a traction substation [2].

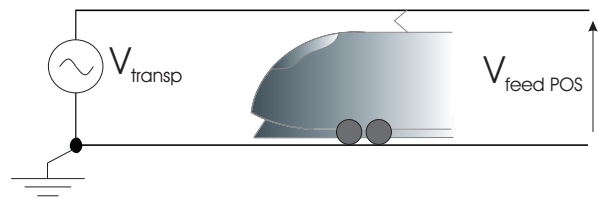

Figure 2: Mono-voltage system configuration. 
These substations are connected between two of the three phases of the highvoltage network. Each of these sectors can use either mono-voltage system $(1 \times 25 \mathrm{kV})$ or bi-voltage system $(2 \times 25 \mathrm{kV})$. In mono-voltage system, the feeding conductors are set to the specified voltage level (see Figure 2).

In bi-voltage systems, a higher voltage is set between feeding conductors [3, 4]. This voltage is reduced by using autotransformers distributed along the catenary (see Figure 3). In these systems, the term cell normally refers to the portion of catenary located between two consecutive autotransformers. Typical values for cell lengths are $10-15 \mathrm{~km}$.

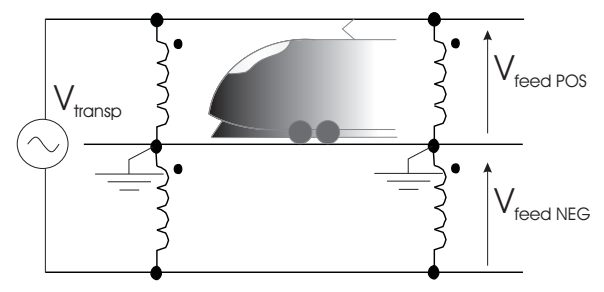

Figure 3: Bi-voltage system configuration.

It has been proven that bi-voltage systems can be represented as if they were mo-voltage $[5,6]$. For that reason, in this paper only mono-voltage systems are considered in the discussions, without loss of generality. Actually, as this paper is focused on driving simulation, the power supply system considered for the discussions is not a critical aspect.

\subsection{Electro-technical dimensioning criteria and driving mode}

Railways power supply systems are dimensioned to be able to supply the power required by the trains, usually assuming a long-term estimation of the traffic needs. From an electro-technical point of view, the power supply system is considered to be able to supply the required power if the following restrictions are fulfilled:

- Voltage in the catenary has to be within the range specified by UIC-600 standard, which specifies the upper and lower limits depending of its duration.

- Currents circulating along the catenary and through transformers (and autotransformers) have to be lower than the rated values, in order to ensure no overheating will occur. These limits are often expressed as power limits instead of current limits.

- Power supplied by the three-phase network through the traction substations is also frequently limited when the network is too weak, in order in order to ensure a proper operation of the network.

Figure 4 shows the train power consumptions of two different driving modes as a function of the train position. In the figure, driving mode 1 and 2 could correspond respectively to a MTD and to a less aggressive driving (lower accelerations and somehow lower speeds). Electrical sections have been 


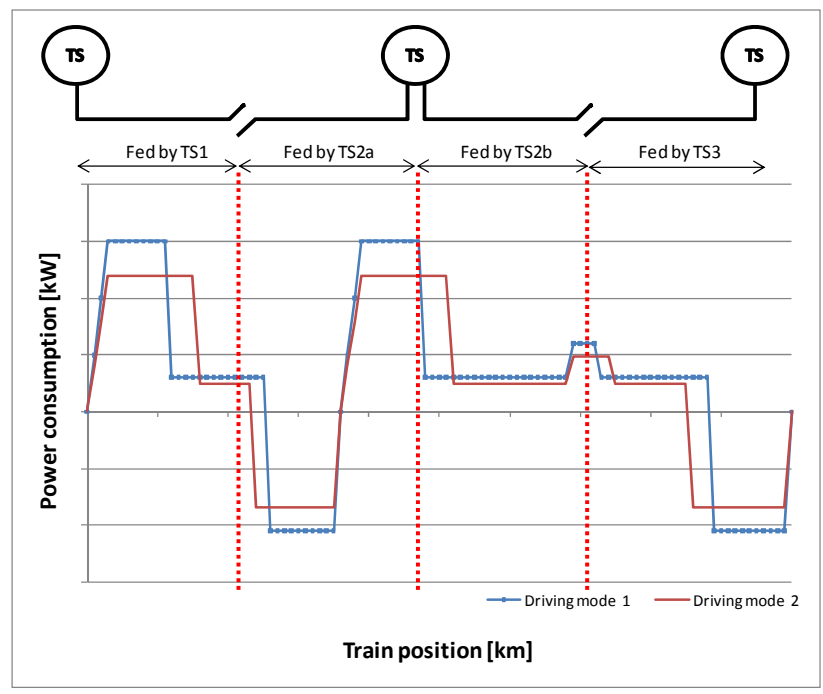

Figure 4: $\quad$ Power consumption comparison of two different driving modes.

represented and each of them is fed with one power transformer (TS1, TS2a, TS2b and TS3), as they are electrically isolated.

The voltage $\mathbf{V}(t, t s)$ at train $t$ pantograph, fed from a substation transformer $t s$ can be calculated using Eq. (1):

$$
\mathbf{V}(t, t s)=\mathbf{V}_{n o-\text { load }}(t s)-\sum_{i \in t s} \mathbf{Z}(t s) \cdot \mathbf{I}(i)-\sum_{i \in t s} \mathbf{Z}_{C A T}^{\prime} \cdot \mathbf{I}(i) \cdot D(i, t s)
$$

where $\mathbf{V}_{\text {no-load }}(t s)$ is the non-load voltage in the substation transformer $t s$, $\mathbf{Z}(t s)$ is the Thevenin equivalent impedance of substation transformer $t s$, $\mathbf{I}(i)$ is the current consumed by a train $i$ fed by substation transformer $t s$, $\mathbf{Z}_{C A T}^{\prime}$ is the per length unit impedance of the catenary and $D(i, t s)$ is the distance between train $t$ and substation transformer .

As shown in Eq. (1), the second term is proportional to currents (which are proportional to power consumptions) but the third one is proportional both to currents and to distances to the substation. When considering the driving modes represented in Figure 4, it cannot be established that MTD (driving mode 1) leads to lower voltages than driving mode 2 , even if peak power consumptions are higher in MTD, because distances from power peaks to substations are just different in both cases.

As the topology of each electrical section is radial, the maximum instant current circulating in the catenary $\mathbf{I}_{C \text {, max }}(t s)$ corresponds normally to the total current supplied by the substation, which is proportional to the supplied power as 
expressed in Eq. (2) (even if exceptions to that rule may occur when regenerated power is high).

$$
\mathbf{I}_{C, \max }(t s)=\sum_{i \in t s} \frac{\mathbf{S}^{*}(i)}{\mathbf{V}^{*}(i, t s)}
$$

In order to evaluate if currents are under the rated values, normally quadratic mean currents are calculated from the instant values.

Also for currents, MTD do not necessarily correspond to the higher values due to two different effects. First, if time is kept constant between two consecutive trains, the distance between two consecutive trains is always higher in MTD than in other driving modes. In other words, the same substation may have to supply power to eventually more trains. Second, as power consumption spatial distributions differ from one driving mode to other, the substations that supply the power (and thus the current) may be different.

Finally, the instant power $\mathbf{S}_{\text {total }}(t s)$ supplied by the substations transformer ts is calculated in Eq. (3)

$$
\mathbf{S}_{\text {total }}(t s)=\mathbf{S}_{\text {loss }}(t s)+\sum_{i \in t s} \mathbf{S}(i)
$$

where $\mathbf{S}_{\text {loss }}(t s)$ represents the power losses supplied by the substation transformer $t s$ and includes losses in the catenary, in the transformer itself and in the three-phase network.

For the same reasons mentioned for currents, MTD is not necessarily the most demanding driving mode for the power supply limits of each substation.

\section{Simulation procedure}

\subsection{Overview}

In order to design the power supply system of a railway network the traffic (including train movement) and the power supply are often supposed to be uncoupled. The design is then done iteratively in two different steps, as shown in Figure 5.

Firstly the traffic is simulated for each discrete time step and a traffic scenario is obtained, usually assuming MTD. A traffic scenario is composed by the list of locations and power consumptions of each train at each time step. Then an electrical simulator, typically solving a power flow for each time step, is used to determine all the voltages, currents and power flows and to determine if the power supply works properly. The design is modified and the electrical simulation is repeated until the power supply works properly. 


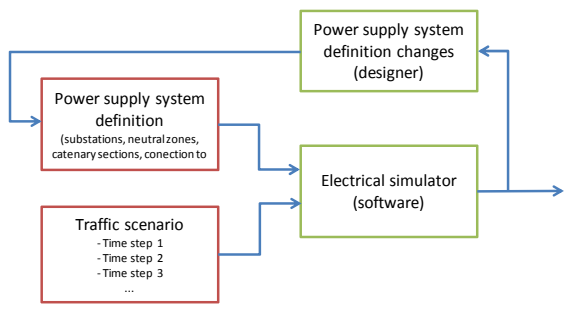

Figure 5: Power supply design process considering deterministic traffic scenario.

Once determined that MTD is not necessarily the most demanding driving mode (neither for voltages, nor for currents, nor for power flows), it is proposed to modify the design process so that the power supply is evaluated with a set of stochastic scenarios (see Figure 6).

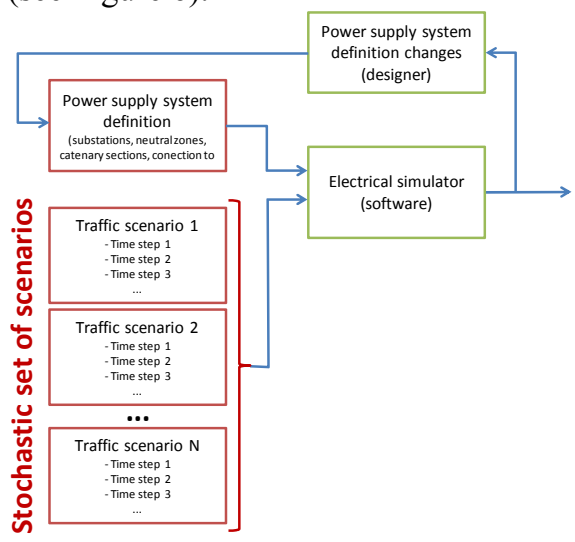

Figure 6: Power supply design process considering stochastic traffic scenarios.

The stochastic traffic generator presented in this paper is intended to provide such a set of scenarios to run a Monte Carlo load flow. To produce these scenarios, well-known MTD simulations have been enriched with the following add-ins: (i) stochastic speed profiles, (ii) random speed reductions and (iii) stochastic stop times.

\subsection{Stochastic traffic generation}

Traffic generation is usually performed in two steps, which are described in the following parts of this section: (i) stochastic minimal time single train driving (single train movement and power consumption calculations) and (ii) stochastic traffic mesh generation. 


\subsubsection{Stochastic minimal time driving}

The stochastic MTD driving simulation calculates the movement of the train between two consecutive stops (position, speed and acceleration) as well as the power consumption o regeneration, for each time step. To determine the movement, Newton's second law (see Eq. (4)) is integrated:

$$
F_{t}-F_{w}-F_{c}-F_{r}=k M \frac{d v}{d t}
$$

where $F_{t}$ is the traction force, $F_{w}$ is the resistant force due to the slope of the railway line, $F_{c}$ is the resistant force due to the curvature of the railway line, $F_{r}$ is the running resistance force, $M$ is the mass of the train, $k$ is a factor that is commonly used to consider the rotating inertia, and $v$ is the speed of the train.

The resistance force due to the slope of the line is proportional to the mass of the train and the slope of the line. The resistance force due to the curvature is also proportional to the mass of the train and inverse to the curvature radius. The running resistance force is normally approximated by a quadratic expression function of the speed. Finally, the traction force $F_{t}$ is decided for each time step in order to fulfil the speed limits (rolling stock limits and infrastructure limits), maximum acceleration/deceleration or other constraints defining the driving mode.

Once the movement of the train has been solved, the electrical consumed power $P_{e}$ and regenerated power $P_{r}$ is determined using Eqs. (5) and (6) respectively:

$$
\begin{gathered}
P_{e}=\frac{F_{t} \cdot v}{\eta_{e, m}}+P_{a n c} \\
P_{r}=F_{t} \cdot v \cdot \eta_{m, e}+P_{a n c}
\end{gathered}
$$

where $\eta_{e, m}$ is the efficiency of the electrical to mechanical power conversion, $\eta_{m, e}$ is the efficiency of the mechanical to electrical power conversion and $P_{a n c}$ is the power consumed by ancillary services (air conditioning, pressure equipment, lights, etc.).

The simulation of each train movement between two consecutive stations is done assuming that starting time and starting station are the temporal and spatial origins. For that reason, once all the train movements have been calculated, the traffic mesh has to be built-up by shifting the individual movements to their space and time origin. To assign time origins, train frequencies and stop times in the stations have to be specified. Once the traffic mesh has been constructed, for each time step all the active trains are identified and the traffic scenario can be constructed. 
As discussed previously, actual traffic can be very different depending on the driving, on the traffic contingencies or even on the number of passengers. For that reason, the following aspects have been modelled:

- Depending on the operational conditions, train driving can be faster or slower, but maximum speed limits have always to be observed. Thus, the speed limit of each section has been assumed to follow a normal probability distribution, which is truncated at the maximum speed limit (see Figure 7).

- In dense traffic situations, due to track occupancy some trains may interfere with other trains, by forcing them to reduce their speed or even making them to stop. In many cases these situations can be identified and eliminated when signalling is designed, but when power supply is being designed no information is normally available. To model this kind of situations, in some cases (randomly selected) an additional speed reduction is added (see Figure 7): their position is assumed to be uniformly distributed along the line and their established speed has been assumed to take discrete values.

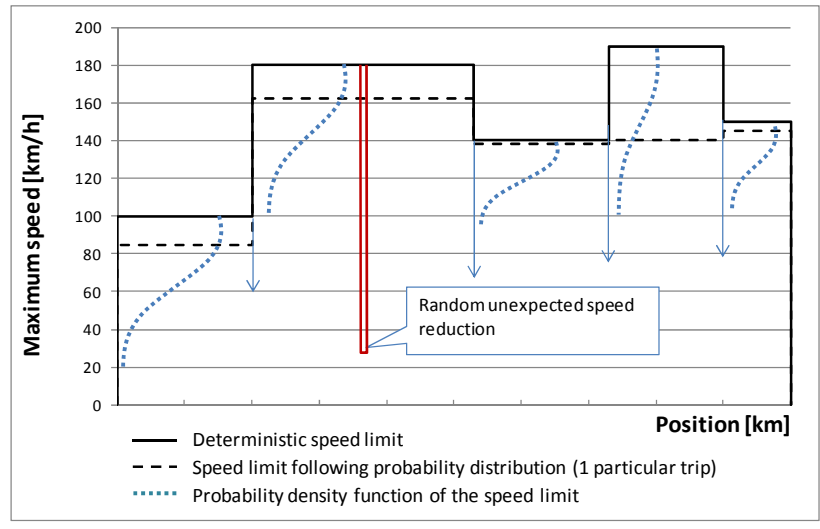

Figure 7: Stochastic speed limits for a single train.

- While in peak hours some stations can be overcrowded and stop time higher, in off-peak hours stop time can be reduced. To model this, the stop time has been considered to follow a normal probability distribution (see Figure 8), whose mean is the rated time stop, truncated in order to have always positive times.

In all these aspects, an additional work has to be done to analyze other probability distributions and to compare them with reality. 


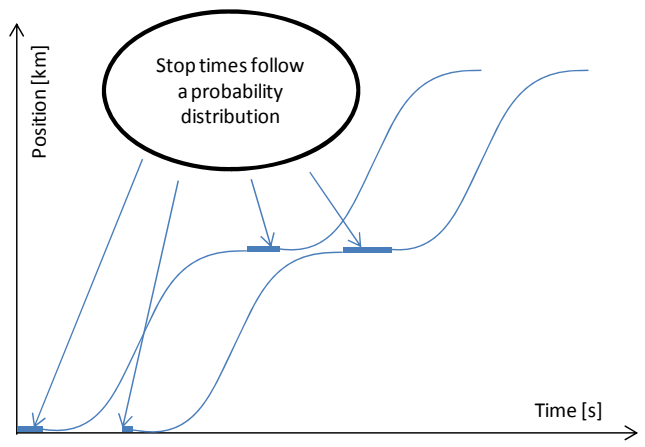

Figure 8: $\quad$ Stochastic stop times when building up the traffic mesh.

\section{Study case}

In order to show the potential of the presented stochastic traffic generator, a 436 $\mathrm{km}$ long section (from Madrid to Lérida) of the Spanish high-speed line MadridBarcelona has been used (its cross-section is shown in Figure 9).

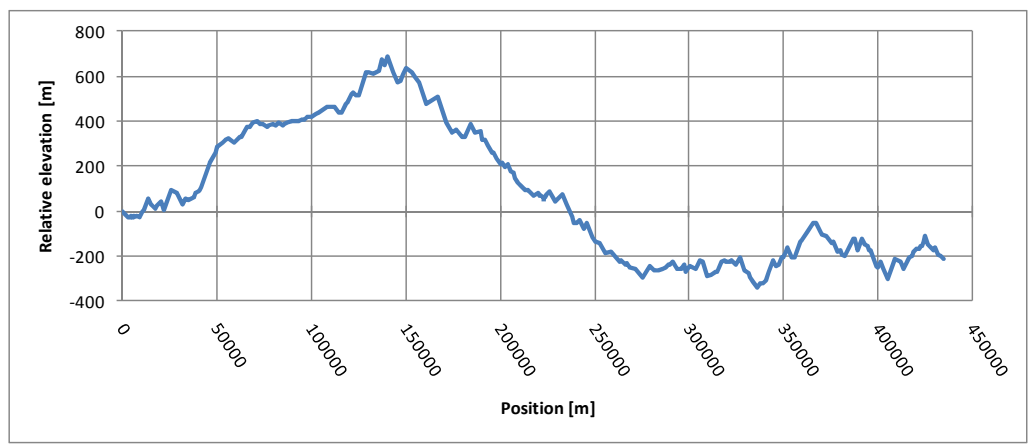

Figure 9: Cross-section of considered part of high-speed line MadridBarcelona.

Generic high-speed trains (410 t heavy, 168 meter long, $850 \mathrm{~kW}$ ancillary services power consumption, without regeneration, $300 \mathrm{~km} / \mathrm{h}$ maximum speed, $280 \mathrm{kN}$ maximum traction force) have been considered. $3 \mathrm{~min}$ intervals have been presumed.

Figure 10 shows, for every position, the power that should be supplied by a substation to a standard section $35 \mathrm{~km}$ long. It has been calculated as the sum of the electrical power required by all the trains within a $35 \mathrm{~km}$ moving window, assuming MTD. This kind of graphics gives a good idea about how does power consumption accumulate along the railway and which parts of the line are more demanding. 


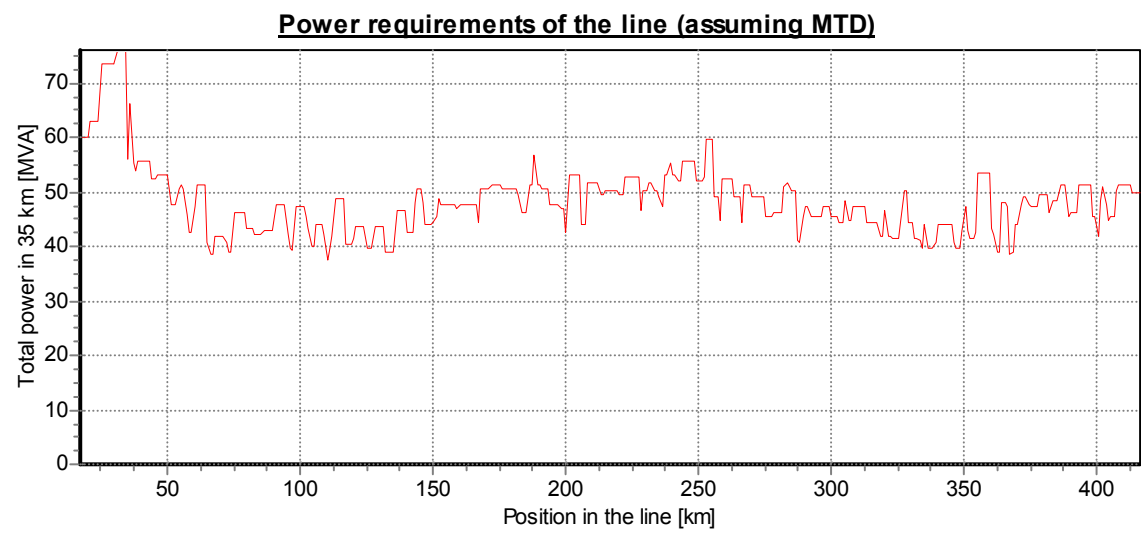

Figure 10: Power requirements considering MTD traffic.

Figure 11 shows the same kind of graphics, but obtained from one of the many scenarios obtained by the stochastic traffic generator. Actual speed limits have been taken as mean values of the normal distributions, with a typical $10 \%$ standard deviation. 3 minutes stop times have been consider, with $30 \mathrm{~s}$ standard deviation. Finally, 20\% steps have been considered for unexpected stops, which occur with a $5 \%$ probability.

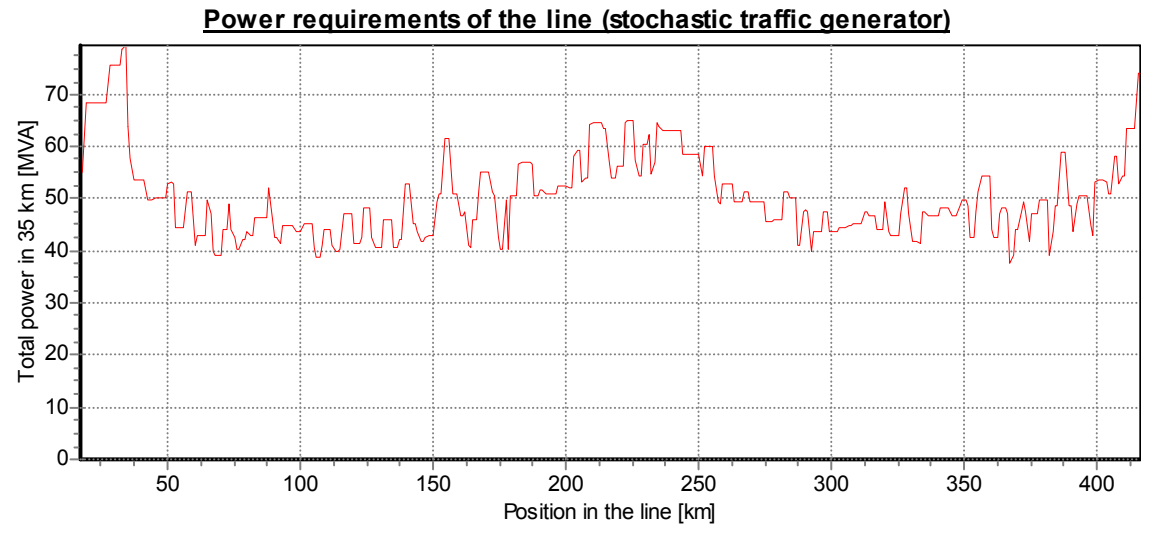

Figure 11: Power requirements considering stochastic traffic (one of the scenarios).

Comparison of Figures 10 and 11 shows that power requirement in both cases are slightly different. Therefore, considering several operation types would be very enriching when carrying out the power supply design. 


\section{Conclusions}

This paper has presented a stochastic traffic generator in which (i) the driving manner is assumed to be stochastic, (ii) random unexpected stops are considered and (iii) the stop time in the stations is also supposed to be stochastic. This simulation tool provides a large number of different driving scenarios to be used for a Monte Carlo load flow analysis for the design of the power supply system.

This stochastic traffic generator has been used to generate traffic scenarios for a section of the Spanish high-speed line Madrid-Barcelona. The power requirement analysis shows that using this kind of tools could lead to more accurate power supply system designs, as more operating conditions can be analyzed.

Future developments should include refining probability distribution functions and validating them with measurements. In addition, the integration of this tool with a power flow tool would ease the penetration of such tools in the industry.

\section{References}

[1] P. Lukaszewicz, "Energy Consumption and Running Time for Trains: modelling of running resistance and driver behaviour based on full scale testing" PhD Thesis. Royal Institute of Technology (KTH). Stockholm, 2001

[2] E. Pilo, L. Rouco, A. Fernández and A. Hernández-Velilla, “A simulation tool for the design of the electrical supply system of high-speed railway lines," in IEEE PES Summer Meeting 2000, Seattle (USA)

[3] P. H. Hsi, S. L. Chen and R. J. Li, "Simulating on-line dynamic voltages of multiple trains under real operating conditions for AC railways," IEEE Transactions on Power Systems, vol. 14, pp. 452-459, 1999

[4] R. J. Hill and I. H. Cevik, "On-line simulation of voltage regulation in autotransformer-fed AC electric railroad traction networks," IEEE Transactions on Vehicular Technology, vol. 42, pp. 365-372, 1993

[5] E. Pilo, L. Rouco and A. Fernández, "A reduced representation of $2 / \mathrm{spl}$ times $/ 25 \mathrm{kV}$ electrical systems for high-speed railways," in IEEE/ASME Joint Rail Conference, Chicago, 2003, pp. 199-205

[6] G. Varju, "Simplified method for calculating the equivalent impedance of an AT system," Innotech Ltd (Innovation Park of Technical University of Budapest), Budapest, July 1996 